\title{
Potassium Monitoring From Multilead T-wave Morphology Changes During Hemodyalisis: Periodic Versus Principal Component Analysis
}

\author{
Flavio Palmieri ${ }^{1,2,3}$, Pedro Gomis ${ }^{1,2}$, José Esteban Ruiz ${ }^{4}$, Dina Ferreira ${ }^{3}$, Alba Martín-Yebra ${ }^{2,5}$, \\ Esther Pueyo $^{2,5}$, Pablo Laguna ${ }^{2,5}$, Juan Pablo Martínez ${ }^{2,5}$, Julia Ramírez ${ }^{6}$ \\ ${ }^{1}$ Centre de Recerca en Enginyeria Biomèdica, Universitat Politècnica de Catalunya, Barcelona, Spain \\ ${ }^{2}$ CIBER en Bioingeniería, Biomateriales y Nanomedicina (CIBER-BBN), Zaragoza, Spain \\ ${ }^{3}$ Laboratorios Rubio, Castellbisbal, Barcelona, Spain \\ ${ }^{4}$ Nephrology Department, Hospital Clínico Universitario Lozano Blesa, Zaragoza, Spain \\ ${ }^{5}$ BSICoS Group, I3A, IIS Aragón, Universidad de Zaragoza, Zaragoza, Spain \\ ${ }^{6}$ William Harvey Research Institute, Queen Mary University of London, London, United Kingdom
}

\begin{abstract}
Background: End-stage renal disease (ESRD) patients undergoing hemodyalisis therapy $(H D)$ experience blood potassium $\left(\left[\mathrm{K}^{+}\right]\right)$variations that are reflected on the $T$ wave (TW) morphology. Methods: We evaluated the performance of different lead space reduction (LSR) methods: principal component analysis (PCA), maximising the $T W$ energy, and two derived versions of periodic component analysis ( $\pi C A$ ) named $\pi C A^{\mathrm{B}}$ and $\pi C A^{\mathrm{T}}$, maximising the QRST or TW beat periodicity. We applied these methods to 12-lead electrocardiogram (ECG) from 24 ESRD-HD patients. Then, we derived three markers of TW morphology changes $\left(d_{w}^{u}, d_{w}\right.$ and $\left.\hat{d}_{w, c}\right)$, comparing an average $T W$ derived every 30 min with that at the $H D$ end, from the PCA, $\pi C A^{\mathrm{B}}$ and $\pi C A^{\mathrm{T}}$ based leads having the highest TW energy content. Similarities between these three methods were assessed by using Bland-Altman plots and the linear fitting error $(\epsilon)$ evaluated from the $12^{\text {th }}$ to the $44^{\text {th }} h$ of ECG recordings after the $H D$ onset. Results: All series of $d_{w}^{u}$, $d_{w}$ and $\hat{d}_{w, c}$ values showed good degree of mutual agreement (median bias $\leq 0.5 \mathrm{~ms}$ ) and a small deviation from linearity in the $\left[\mathrm{K}^{+}\right]$increasing stage (median $\epsilon \leq 3.3 \mathrm{~ms}$ ). Conclusions: PCA and $\pi C A$ can be used interchangeably to track TW changes in ESRD-HD patients, in this type of low noise contamination ECG recordings.
\end{abstract}

\section{Introduction}

Cardiovascular diseases are the major causes of morbidity and mortality in end-stage renal disease (ESRD) patients undergoing hemodialysis (HD). In particular, variations in blood potassium concentration $\left(\Delta\left[K^{+}\right]\right)$, among other effects, can alter the spatio-temporal ventricular re- polarization sequence, reflected as T-wave (TW) morphology changes on the electrocardiogram (ECG) [1], which may set the stage for lethal cardiac arrhythmia. In a previous study [2], we investigated the ability of time-warpingbased markers of TW morphology changes [3] in monitoring $\Delta\left[K^{+}\right]$in ESRD-HD patients. Three of these markers showed the highest correlation with $\Delta\left[K^{+}\right], d_{w}^{u}$ (implemented as proposed in [3]), $d_{w}$ (a signed version of $d_{w}^{u}$ ), and $\hat{d}_{w, c}$ (a heart rate-corrected version of $d_{w}^{u}$ ). In both studies [2,3], principal component analysis (PCA) was applied to concentrate the TW energy across leads in one single lead, the first principal component (PC1), assuming it was the one with maximal TW energy.

In Monasterio et al. [4] periodic component analysis $(\pi \mathrm{CA})$, an eigenvalue-based transformation aiming to emphasise periodicity in multi-channel signals, was used as an alternative to PCA to emphasise the alternant component in TW corrupted by high noise contamination. The study proved the superior performance of $\pi \mathrm{CA}$ as compared to PCA for alternans detection. The objective of this work is to compare the performance of PCA and $\pi \mathrm{CA}$ as lead space reduction (LSR) step previous to time-warping analysis for $\Delta\left[K^{+}\right]$monitoring in HD. In particular, the $\pi \mathrm{CA}$ version proposed in [4] for TW alternans (periodicity every two beats) analysis was here modified to study TW morphology (periodicity every beat). Then, time-warping was performed over the PCA- and $\pi$ CA-transformed leads, extracting and comparing $d_{w}^{u}, d_{w}$ and $\hat{d}_{w, c}$ series.

\section{Study Population and Protocol}

A standard, 12-lead, 48-hour ECG Holter recording (H12+, Mortara Instruments, sampling frequency 1 $\mathrm{kHz}$, amplitude resolution $3.75 \mu \mathrm{V}$ ), was recorded from 24 ESRD-HD patients at Hospital Clínico Universitario 

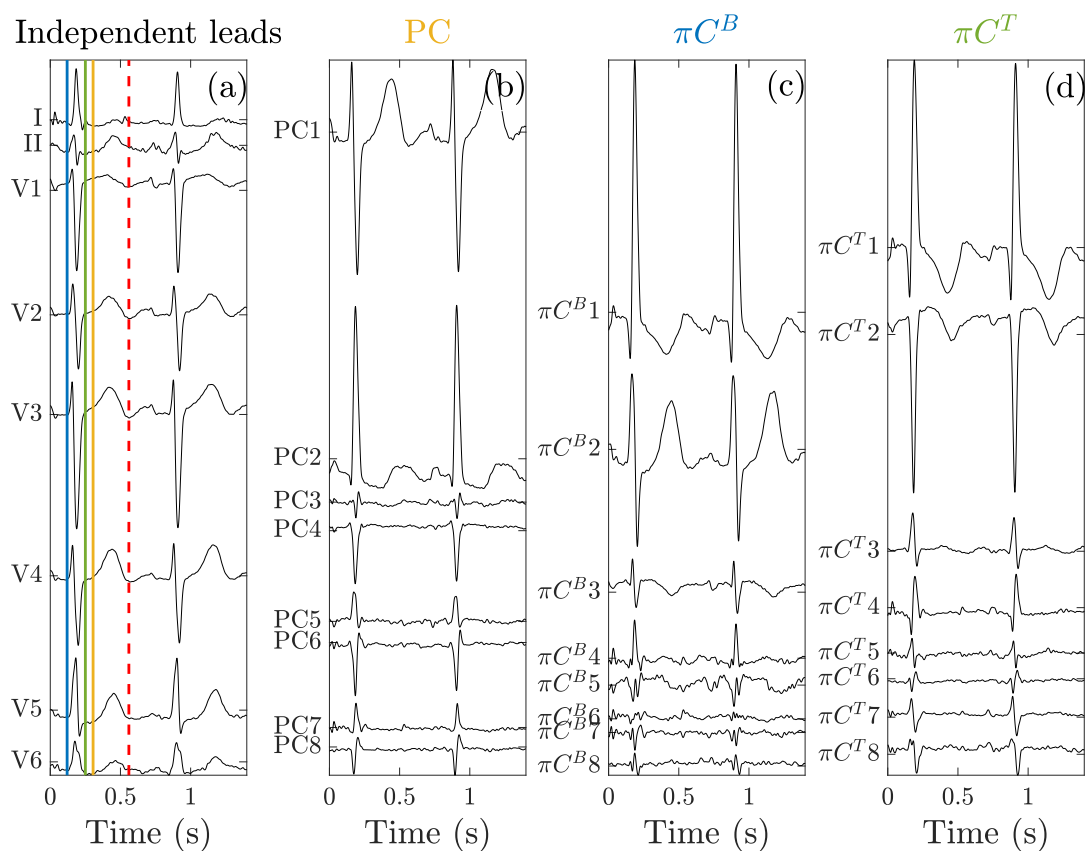

Figure 1: Panel (a) shows 8 independent leads from one of the patients. Each vertical solid line represents the starting point of the window containing the samples used to build the eigenvectors in PCA (orange, denoting Ton), $\pi C A^{\mathrm{B}}$ (blue, denoting QRSon) and $\pi C A^{\mathrm{T}}$ (green, denoting QRSon+130ms). The vertical red dashed line marks the end of the window (TW end). The resulting PCs, $\pi C^{\mathrm{B}} \mathrm{s}$ and $\pi C^{\mathrm{T}} \mathrm{s}$ are depicted panel (b) to (d) respectively.

Lozano Blesa (Zaragoza, Spain) starting 5 minutes before the HD session. The study protocol was approved by the ethical committee (CEICA, ref. PI18/003) and all patients signed informed consent.

\section{Methods}

ECG pre-processing: ECG pre-processing included low-pass filtering ( $40 \mathrm{~Hz}$ cut-off) to remove muscular and power-line noise and high-pass filtering ( $0.5 \mathrm{~Hz}$ cut-off) to attenuate baseline wander. QRS complexes were detected and delineated in each single lead using a wavelet-based delineation method [5].

Lead Space Reduction: A transformation matrix $\Psi$ was estimated for each LSR method and then applied to the 8 independent leads to obtain their corresponding transformed leads (PCs or $\pi \mathrm{Cs}$ ).

Principal Component Analysis: The coefficients defining $\boldsymbol{\Psi}_{\mathrm{PCA}}$ were obtained from the eigenvectors of the $8 \times 8$ inter-lead auto-correlation matrix computed using the samples in the TW defined from T-on to T-off (from orange to red dashed line in Fig. 1(a)) [3] in a 10-min window at the end of the HD session. As a result, PC1 was the transformed lead that maximised the TW energy, as in Fig. 1 (b).

Periodic Component Analysis: QRST complexes, defined as a window of $450 \mathrm{~ms}$ from the QRS onset (blue line in Fig. 1(a)), were taken from the above mentioned 10-min window for the transformation learning, resulting in the $\pi C^{\mathrm{B}} \mathrm{s}$ (Fig. 1(b)). Alternatively, considering that the QRS complex and TW, even both being one-beat periodic, do not necessarily have the same spatial distribution, only the TW area was considered to calculate the eigenvectors using a window from the QRS onset plus $130 \mathrm{~ms}$ to $450 \mathrm{~ms}$ (green to red dashed line in Fig. 1(a)), resulting in $\pi C^{\mathrm{T}} \mathrm{s}$ (Fig. 1(d)). Let $m=1$ be the one-beat periodicity, $K$ the number of heartbeats in the analysing window, $N$ the number of samples in the selected beat interval, $L$ the number of leads and $x_{k, l}(n)$ the $n^{t h}$ sample in the $k^{t h}$ heartbeat for a given $l^{\text {th }}$ lead. In vector notation each QRST complex, or $\mathrm{TW}$, was defined as $\mathbf{x}_{k, l}=\left[x_{k, l}(0) \cdots x_{k, l}(N-1)\right]^{\mathrm{T}}$. To avoid extra variability due to the single-lead delineation, a pre-alignment of the selected intervals in each lead was performed by computing a reference QRST complex (or TW) as the mean of all QRST complexes (or TW) in that particular window. Then, cross-correlation was computed between the reference and each QRST complex (or TW) and these were shifted according to the lag of maximum cross-correlation. For each heartbeat, all leads were put together in the $L \times N$ matrix:

$$
\mathbf{X}_{k}=\left[\mathbf{x}_{k, 1} \cdots \mathbf{x}_{k, L}\right]^{\mathrm{T}}
$$

Two matrices $\mathbf{X}$ and $\mathbf{X}^{(m)}$ were constructed by concatenating $\mathbf{X}_{k}$ from 1 to $K$ and from $1+m$ to $K+m$, respec- 
tively:

$$
\mathbf{X}=\left[\mathbf{X}_{1} \cdots \mathbf{X}_{K}\right]^{\mathrm{T}} ; \mathbf{X}^{(m)}=\left[\mathbf{X}_{1+m} \cdots \mathbf{X}_{K+m}\right]^{\mathrm{T}}
$$

Then the spatial correlation matrices $\mathbf{R}_{\mathbf{X}}$ for $\mathbf{X}$ and $\mathbf{A}_{\mathbf{X}^{(m)}}$ for $\left(\mathbf{X}^{(m)}-\mathbf{X}\right)$ were computed as:

$$
\begin{gathered}
\mathbf{R}_{\mathbf{X}}=\frac{1}{K N} \mathbf{X X}^{\mathrm{T}} \\
\mathbf{A}_{\mathbf{X}^{(m)}}=\frac{1}{K N}\left(\mathbf{X}^{(m)}-\mathbf{X}\right)\left(\mathbf{X}^{(m)}-\mathbf{X}\right)^{\mathrm{T}}
\end{gathered}
$$

Finally, $\Psi_{\pi \mathrm{CA}}$ was chosen as the generalised eigenvector matrix of the pair $\left(\mathbf{A}_{\mathbf{X}^{(m)}}, \mathbf{R}_{\mathbf{X}}\right)$ [4], with the eigenvectors sorted according to the corresponding eigenvalues in ascending order of magnitude, being $\pi \mathrm{C} 1$ the spatial direction in which periodic signal component is better seen.

Time-warping TW morphology quantification: For each patient and transformation, all TW in the first and the second transformed leads were delineated [5]. In the case of PCA and $\pi C^{\mathrm{T}}$, the first component was chosen for analysis, since, by its construction, it always has the maximum TW energy. However, for $\pi C^{\mathrm{B}}$, since QRS is included in the transformation learning, there is no guarantee that the TW is maximised in the first transformed lead. Therefore, to select the $\pi C^{\mathrm{B}}$ lead with the maximum TW energy, the TW energy was computed in the first two $\pi \mathrm{C}^{\mathrm{B}} \mathrm{s}$ in the learning 10-min window at the HD end-stage, and the one with the maximum energy was chosen. Then, TW in the chosen transformed leads were selected in a 2-min window centred on the $5^{t h}$ and $35^{t h}$ minute of each available hour and used to compute a mean warped TW (MWTW) as in [3]. That window is narrow enough to allow the assumption of stability in heart rate $(\mathrm{HR})$ and $\left[\mathrm{K}^{+}\right]$levels. The MWTW at the end of the HD was taken as the reference and the morphology of each MWTWs was compared with respect to it performing TW time-warping analysis as described in $[2,3]$. The resulting marker $d_{w}^{u}$ quantifies the level of warping needed to optimally align two TW as:

$$
d_{w}^{u}=\frac{1}{N_{r}} \sum_{n=1}^{N_{r}}\left|\gamma^{*}\left(t^{r}(n)\right)-t^{r}(n)\right|
$$

being $\gamma^{*}\left(\mathbf{t}^{r}\right)$ the optimal warping function that minimises the amplitude difference between the square-root slope function [6] of the reference MWTW $\boldsymbol{f}^{r}\left(\boldsymbol{t}^{r}\right)=$ $\left[f^{r}\left(t^{r}(1)\right), \ldots, f^{r}\left(t^{r}\left(N_{r}\right)\right)\right]^{\mathrm{T}}$ and each available MWTW $\boldsymbol{f}^{s}\left(\boldsymbol{t}^{s}\right)=\left[f^{s}\left(t^{s}(1)\right), \ldots, f^{s}\left(t^{s}\left(N_{s}\right)\right)\right]^{\mathrm{T}}$; with $\boldsymbol{t}^{r}=$ $\left[t^{r}(1), \ldots, t^{r}\left(N_{r}\right)\right]^{\mathrm{T}}$ and $\boldsymbol{t}^{s}=\left[t^{s}(1), \ldots, t^{s}\left(N_{s}\right)\right]^{\mathrm{T}}$ and $N_{r}$ and $N_{s}$ are the total duration of $\boldsymbol{t}^{r}$ and $\boldsymbol{t}^{s}$. The original definition of $d_{w}^{u}$ [3] was modified [2] to allow the marker to be signed, therefore distinguishing TW widening from narrowing. The signed $d_{w}$ and its sign, $s_{d}$, are:

$$
d_{w}=\left(\frac{s_{d}}{\left|s_{d}\right|}\right) \frac{1}{N_{r}} \sum_{n=1}^{N_{r}}\left|\gamma^{*}\left(t^{r}(n)\right)-t^{r}(n)\right|
$$

$$
s_{d}=\sum_{n \in N_{r}^{u}}\left(\gamma^{*}\left(t^{r}(n)\right)-t^{r}(n)\right)+\sum_{n \notin N_{r}^{u}}\left(t^{r}(n)-\gamma^{*}\left(t^{r}(n)\right)\right)
$$

with $N_{r}^{u}$ being the set of TW up-slope samples. A positive sign means that the $\boldsymbol{f}^{s}\left(\boldsymbol{t}^{s}\right)$ has to be widened to fit the $\boldsymbol{f}^{r}\left(\boldsymbol{t}^{r}\right)$ and vice-versa for a negative sign. Finally, to compensate for concomitant TW variations due to $\mathrm{HR}, d_{w}$ was modelled as the sum of two components: $d_{w}=d_{w, c}+d_{w, \mathrm{HR}}$ where $d_{w, \mathrm{HR}}$ accounts for TW morphology changes related to HR, and $d_{w, c}$ accounts for other changes, including those related to $\Delta\left[K^{+}\right]$. To estimate $d_{w, c}$, we used the following correction formula, based on a linear approximation used to account for the HR dependency of the QT interval as in [7]:

$$
\hat{d}_{w, c}=d_{w}-\hat{c}\left(\mathrm{RR}_{\mathrm{s}}-\mathrm{RR}_{\mathrm{r}}\right)
$$

being $\hat{d}_{w, c}$ the estimated $d_{w, c}, \mathrm{RR}_{\mathrm{s}}$ and $\mathrm{RR}_{\mathrm{r}}$ are the mean RR interval from the $s^{\text {th }}$ studied and the reference segments, respectively, and $\hat{c}$ is estimated for each patient [2]. Marker dynamics evaluation: To assess the agreement between LSR-specific marker, Bland-Altman (B-A) plots evaluated for each patient and each pair of transformations were used, extracting the bias and confidence limits (CL). For every patient, a fitting error $(\epsilon)$ from a linear regression between the $12^{\text {th }}$ and $44^{\text {th }} \mathrm{h}$ after HD onset was computed for each LSR-specific marker. This $\epsilon$ provides information on the marker's deviation from a gradual linear trend along time, which could be related to the trend followed by $\left[\mathrm{K}^{+}\right]$.

\section{Results and Discussion}

The average duration of the ECG recordings was 44 hours, not 48 as expected, mainly due to electrode detachment or early battery exhaustion. $\pi C^{\mathrm{B}} 1$ was chosen in 17 patients $(71 \%)$ as the one with the maximum TW energy. This would suggest that, for $29 \%$ of patients, the spatial projection that maximises the QRST periodicity was not the same that maximises the TW periodicity.

The evolution of the PCA- and $\pi$ CA-based $d_{w}^{u}$, along the ECG recording is shown in Fig. 2 (a) and similar trends were found also for $d_{w}$ and $\hat{d}_{w, c}$ markers (not depicted). A detailed view of the first 10 hours is also presented (median values only). In this specific interval, all the markers show a V-shaped curve having the vertex at the end of the HD (Fig. 2 (b)), similarly to the reported $\left[K^{+}\right]$-recovery curves $[8,9]$. This highlights an interesting analogy that needs to be further investigated.

Looking at the results in Table 1, the bias and CL were, in median, consistently smaller when comparing PC with $\pi C^{\mathrm{T}}(-0.2 \leq \mathrm{B}-\mathrm{A}$ bias $\leq 0.0$ and $7.5 \leq \mathrm{CL} \leq 9.3)$, and in both cases smaller $\epsilon$ (being $1.8 \leq \epsilon \leq 2.1$ for PC and $2.0 \leq \epsilon \leq 2.4$ for $\pi C^{\mathrm{T}}$ ) than $\pi C^{\mathrm{B}}$. Results for B-A bias and $\mathrm{CL}$ indicate that the agreement is similar between $\pi C^{\mathrm{T}}$ and 
PCA. However, since PCA separates signal components according to their variance, projecting the component with the highest energy onto the first transformed lead could be a limitation in highly noisy ECG recordings [4].

From the $10^{t h} \mathrm{~h}$ on, the abrupt changes of markers median values gradually reduce, settling themselves to a trend that could be qualified as linear. By assuming that, a small median $\epsilon$ for a given LSR technique would suggest that it resulted in more coherent measurements across patients for a specific marker. However, even if $\epsilon$ values in Table 1 may indicate at PCA as the technique leading to the least scattered markers, the median and IQR are very similar to each other to definitively conclude which among PCA, $\pi C^{\mathrm{T}}$ and $\pi C^{\mathrm{B}}$ was the most consistent in terms of measurements.

\section{Conclusions}

In this work, we tested the performance of $\pi \mathrm{CA}$-based and PCA LSR methods, by comparing three TW timewarping based markers computed over long duration ECG recordings from 24 ESRD-HD patients. Our results would support the interchangeable usage of both PCA and $\pi \mathrm{CA}$ as preprocessing steps to track TW morphology variations during and after HD. Future studies under heavier noise conditions will elucidate if $\pi C^{\mathrm{T}}$ is preferred over $\pi C^{\mathrm{B}}$ or PCA, as shown in $\mathrm{T}$ wave alternans detection [4].

Table 1: Median bias and confidence limits, CL from B-A plot and meadian $\epsilon / \mathrm{IQR}$ evaluated across patients.

\begin{tabular}{|l|c|c|c||c|c|c|}
\hline \hline & \multicolumn{2}{|c||}{ median\{B-A bias $\} / \mathrm{CL}(\mathrm{ms} / \mathrm{ms})$} & \multicolumn{3}{c|}{ median $\{\epsilon\} / \mathrm{IQR}(\mathrm{ms} / \mathrm{ms})$} \\
\cline { 2 - 7 } & $\mathrm{PC}-\pi C^{\mathrm{B}}$ & $\mathrm{PC}-\pi C^{\mathrm{I}}$ & $\pi C^{\mathrm{B}}-\pi C^{\mathrm{I}}$ & $\mathrm{PC} 1$ & $\pi C^{\mathrm{B}} \mathrm{s}$ & $\pi C^{1}$ \\
\hline$d_{w}^{u}$ & $0.0 / 11.2$ & $0.0 / 7.5$ & $0.3 / 9.2$ & $1.8 / 1.8$ & $2.4 / 2.0$ & $2.0 / 1.3$ \\
$d_{w}$ & $0.1 / 13.3$ & $-0.1 / 9.5$ & $-0.2 / 14.5$ & $2.2 / 2.6$ & $3.1 / 3.8$ & $2.4 / 2.1$ \\
$\hat{d}_{w, c}$ & $0.5 / 12.8$ & $-0.2 / 9.3$ & $-0.3 / 14.2$ & $2.1 / 1.7$ & $3.3 / 3.4$ & $2.2 / 2.0$ \\
\hline
\end{tabular}

\section{Acknowledgments}

This work was funded by Products \& Technology S.L. (Castellbisbal, Barcelona, Spain), by AGAUR, Generalitat de Catalunya (Spain) grant DI001-2018. The work was also supported by Gobierno de Aragón (project LMP124-18 and reference Group Biomedical Signal Interpretation and Computational Simulation (BSICoS) T39-20R) cofunded by FEDER 2014-2020 "Building Europe from Aragón", by European Research Council (ERC) through project ERC-StG 638284 and by Ministerio de Ciencia e Innovación through projects PID2019-105674RB-I00 and PID2019-104881RB-I00. J. R. would like to thank the support from the Marie Sklodowska-Curie grant agreement No 786833.

\section{References}

[1] Secemsky E, et al. High prevalence of cardiac automonic dysfunction and T-wave alternans in dialysis patients. Heart Rhythm 2011;8(4):592-98.

[2] Palmieri F, et al. Monitoring blood potassium concentration in hemodialysis patients by quantifying T-wave morphology dynamics. Ann Biomed Eng 2020 submitted;.
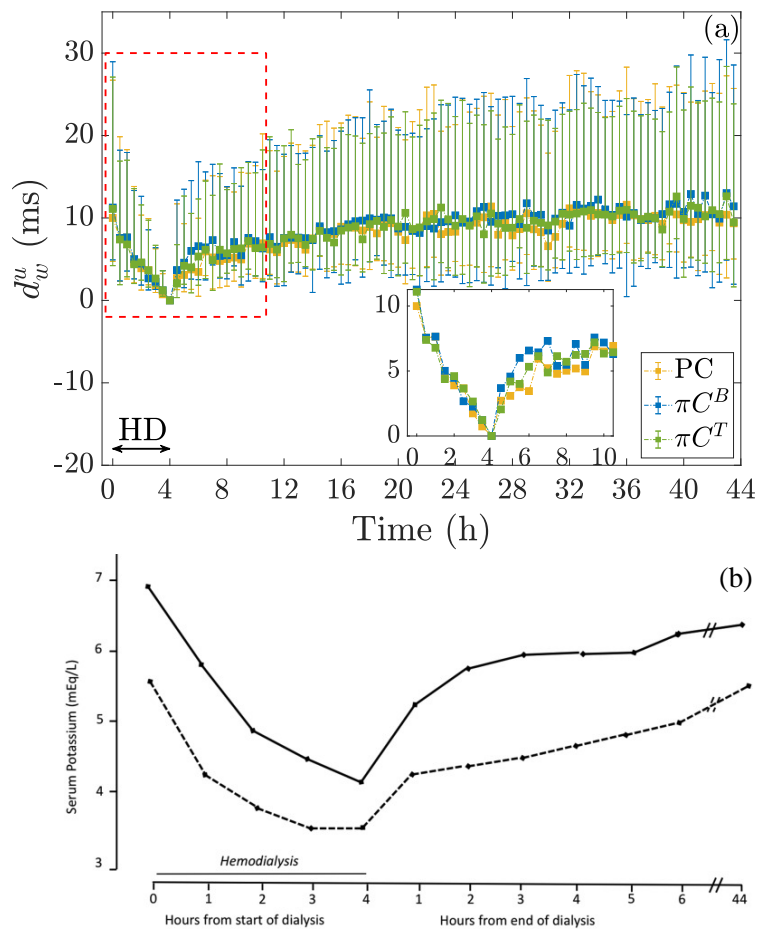

Figure 2: Time dynamics for each marker (a), expressed as median and IQR. A detailed view of the first 10 hours (red dashed rectangle) is also shown to allow its comparison with the $\left[\mathrm{K}^{+}\right]$-recovery curves available the in literature (panel (b)) from [9] Fig.1, p. 3442.

[3] Ramírez J, et al. Variability of ventricular repolarization dispersion quantified by time-warping the morphology of the T-wave. IEEE Trans Biomed Eng 2017;64(7):1619-30.

[4] Monasterio V, et al. A multilead scheme based on periodic component analysis for T-wave alternans analysis in the ECG. Ann Biomed Eng 2010;38(8):2532—-41.

[5] Martínez JP, et al. A wavelet-based ECG delineator: Evaluation on standard databases. IEEE Trans Biom Eng 2004; 4(51):570-81.

[6] Tucker JD, et al. Generative models for functional data using phase and amplitude separation. Comput Statist Data Anal 2013;61:50-60.

[7] Pueyo E, et al. Characterization QT interval adaptation to RR interval changes and its use as a risk-stratifier of arrhytmic mortality in amiodarone-treated survivors of acute myocardial infarction. IEEE Trans Biom Eng 2004;51(9):1511-20.

[8] Blumberg A, et al. Plasma potassium in patients with terminal renal failure during and after haemodialysis; relationship with dialytic potassium removal and total body potassium. Nephrol Dial Transplant 1997;12(8):1629-34.

[9] Pun PH, et al. Dialysate potassium, dialysate magnesium, and hemodialysis risk. J Am Soc Nephrol 2017; 28(12):3441- 51 .

Address for correspondence:

Flavio Palmieri; Universitat Politècnica de Catalunya, Av. Diagonal 647, Barcelona 08028 Spain. flavio.palmieri@upc.edu 